Ibn Al-Haitham Jour. for Pure \& Appl. Sci. 34 (1) 2021

Ibn Al Haitham Journal for Pure and Applied Science

Journal homepage: http://jih.uobaghdad.edu.iq/index.php/j/index

\title{
Topological Structure of Generalized Rough Graphs
}

\section{Samah Sarmad}

\section{Yousif Yaqoub Yousif}

Department of Mathematics, College of Education for Pure Sciences (Ibn Al-Haitham), University of

Baghdad, Baghdad, Iraq.

samahsarmad0@gmail.com

yoyayousif@yahoo.com

Article history: Received 2, February 2020, Accepted20, Febraury,2019, Published in January 2021

Doi: 10.30526/34.1.2559

\begin{abstract}
The main purpose of this paperis to introduce a topological space $\left(D, \tau_{D}\right)$, which is induced by reflexive graph and tolerance graph $D$, such that $D$ may be infinite. Furthermore, we offer some properties of $\left(D, \tau_{D}\right)$ such as connectedness, compactness, Lindelöf and separate properties. We also study the concept of approximation spaces and get the sufficient and necessary condition that topological space is approximation spaces.
\end{abstract}

Keywords. Reflexive graph, tolerance graph, transmitting expression, approximation spaces.

\section{Introduction}

Graph theory [1] is a tool for optimization and solving practical application in all fields such as engineering study and representation of economic and social networks, complex general systems, information theory and others. In particular, graphs are one of the prime objects of study in mathematics.

Rough set was offered by Pawlak [2] as a method for dealing with uncertainly of imprecise data, the equivalence relation is the cornerstone of Pawlak's theory of rough set. Topology is a major mathematics branch with independent theoretic frame work and wide applications.

Z. Li [3] offered the concept of transmitting expression of relation and produced several important results of rough sets topological properties. We can apply topological approaches to the theory of rough set and search the connection between rough set theory and topological theory. The topological properties of various rough operators have been debated in [4]. We built on some of the results in [5-10], [11-15] and [16]. 


\section{Generalized Rough Graphs Generated By Graphs.}

We will remember several fundamental concepts of the theory of rough set. In this article, $D=(V(D), E(D))$ is a graph where $V(D)$ implies the universe which may be infinite, the power set of $V(D)$ symbolized by $P(V(D))$ and the closure of subgraph $Q$ in $D$ symbolized by $\bar{Q}$ wherever $V(D)$ is a topological space.

Let $D=(V(D), E(D))$ be a graph. For each subgraph $Q$ of $D$, we will define operators $D_{-}$and $D_{+}$from $P(V(D))$ to itself as the following:

$$
\begin{aligned}
& D_{-}(Q)=\{\mathrm{r} \in V(D): \text { if }(\mathrm{r}, u) \in E(D), \text { then } u \in V(Q)\}, \\
& D_{+}(Q)=\{\mathrm{r} \in V(D): \text { there exists } u \in V(Q) \text { such that }(\mathrm{r}, u) \in E(D)\} .
\end{aligned}
$$

$D_{-}(Q)$ is named lower approximation of $Q$ and $D_{+}(Q)$ is named upper approximation of $V(Q)$. The pair $(V(D), E(D))$ is named generalized rough graph or generalized approximation space. $Q$ is named generalized exact graph or definable graph if $D_{-}(Q)=D_{+}(Q)$. While $Q$ is called undefinable graph if $D_{-}(Q) \neq D_{+}(Q)$. If $D$ is an equivalence graph, a generalized rough graph $(V(D), E(D))$ means the rough graph in the Pawlak's sense.

Definition 2.1. Let $D=(V(D), E(D))$ be a nonempty graph. We define $\tau_{D}$, for each $Q \subseteq D$ by

$$
\tau_{D}=\left\{Q \subseteq D: D_{-} Q=V(Q)\right\} .
$$

If $D$ is a reflexive graph, then $\tau_{D}$ constitutes a topology on $V(D), \tau_{D}$ can be named the topology produced by $D$.

Definition 2.2. If $D$ is a reflexive graph, then $\left(D, \tau_{D}\right)$ is named the topological space produced by $D$.

Definition 2.3. Let $D$ be a graph, if $D$ is both reflexive and symmetric graph then $D$ is called tolerance graph.

Definition 2.4. Let $D_{\alpha}$ and $D_{\beta}$ be two graphs on $V\left(D_{\alpha}\right)=V\left(D_{\beta}\right)=V(D)$. $D_{\beta}$ is named transmitting expression of $D_{\alpha}$, if for everyғ, $u \in V(D),(\mathrm{r}, u) \in E\left(D_{\beta}\right)$ if and only if $(\mathrm{f}, u) \in$ $E\left(D_{\alpha}\right)$ or there exists $\left\{v_{1}, v_{2}, v_{3}, \ldots, v_{n}\right\} \subseteq V(D)$ where $\left(\mathrm{f}, v_{1}\right) \in E\left(D_{\alpha}\right),\left(v_{1}, v_{2}\right) \in E\left(D_{\alpha}\right), \ldots$, $\left(v_{n}, u\right) \in E\left(D_{\alpha}\right)$.

Proposition 2.5. Let $D_{\alpha}$ be a graph and $D_{\beta}$ the transmitting expression of $D_{\alpha}$, then $D_{\beta}$ is a transitive graph, furthermore,

(1) If $D_{\alpha}$ is reflexive, then $D_{\beta}$ is also reflexive,

(2) If $D_{\alpha}$ is symmetric, then $D_{\beta}$ is also symmetric,

(3) If $D_{\alpha}$ is transitive, then $D_{\beta}=D_{\alpha}$.

Proof. (1) Let $D_{\alpha}$ is reflexive graph, then for each $\mathrm{f} \in V(D),(\mathrm{f}, \mathrm{r}) \in E\left(D_{\alpha}\right)$, since $D_{\beta}$ is a transmitting expression of $D_{\alpha}$, then $(\mathrm{f}, \mathrm{r}) \in E\left(D_{\beta}\right)$, so $D_{\beta}$ is a reflexive graph. 
(2) Let $(f, u) \in D_{\beta}$, since $D_{\beta}$ the transmitting expression of $D_{\alpha}$, then $(r, u) \in D_{\alpha}$ or there exists $\left\{v_{1}, v_{2}, v_{3}, \ldots, v_{n}\right\} \subseteq V(D)$ where $\left(f, v_{1}\right) \in D_{\alpha},\left(v_{1}, v_{2}\right) \in D_{\alpha}, \ldots,\left(v_{n}, u\right) \in D_{\alpha}$ if and only if $(\mathrm{r}, u) \in D_{\beta}$, because $D_{\alpha}$ is symmetric, so $(u, \mathrm{r}) \in D_{\alpha}$ or there exists $\left\{v_{1}, v_{2}, v_{3}, \ldots, v_{n}\right\} \subseteq V(D)$ where $\left(u, v_{n}\right) \in D_{\alpha}, \ldots,\left(v_{2}, v_{1}\right) \in D_{\alpha},\left(v_{1}, \mathbf{r}\right) \in D_{\alpha}$ if and only if

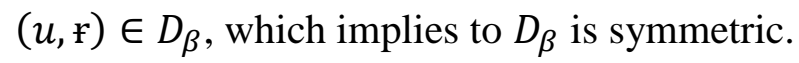

(3) Let $(r, u) \in E\left(D_{\beta}\right)$, we have to show that $(f, u) \in E\left(D_{\alpha}\right)$. Since $D_{\beta}$ the transmitting expression of $D_{\alpha}$, then $(\mathrm{r}, u) \in E\left(D_{\beta}\right)$ if and only if $(\mathrm{f}, u) \in D_{\alpha}$ or there exists $\left\{v_{1}, v_{2}, v_{3}, \ldots, v_{n}\right\} \subseteq V(D)$ where $\left(\mathrm{f}, v_{1}\right) \in E\left(D_{\alpha}\right),\left(v_{1}, v_{2}\right) \in E\left(D_{\alpha}\right),\left(v_{2}, v_{3}\right) \in E\left(D_{\alpha}\right) \ldots$, $\left(v_{n}, u\right) \in E\left(D_{\alpha}\right)$.

(i) If $(f, u) \in E\left(D_{\alpha}\right)$ the prove is complete.

(ii) If there exists $\left\{v_{1}, v_{2}, v_{3}, \ldots, v_{n}\right\} \subseteq V(D)$ where $\left(\mathrm{f}, v_{1}\right) \in E\left(D_{\alpha}\right), \quad\left(v_{1}, v_{2}\right) \in E\left(D_{\alpha}\right)$, $\left(v_{2}, v_{3}\right) \in E\left(D_{\alpha}\right) \ldots,\left(v_{n}, u\right) \in E\left(D_{\alpha}\right)$, and we have that $D_{\alpha}$ is transitive, so $(\mathrm{f}, u) \in E\left(D_{\alpha}\right)$, which means $D_{\beta}=D_{\alpha}$.

Definition2.6. (1) Let $\left(D, \tau_{D}\right)$ be a topological space and $\mathcal{B}_{D}$ a base of $\left(D, \tau_{D}\right)$, where $\left(D, \tau_{D}\right)$ is induced by a reflexive graph $D$. Then $\mathrm{B} \in \mathcal{B}_{D}$ is called maximal element of $\mathcal{B}_{D}$ if does not exist $B^{l} \in \mathcal{B}_{D} \backslash\{B\}$ such that $B \subseteq B^{l}$.

(2)The set of all maximal elements of $\mathcal{B}_{D}$ symbolized by $\mathcal{B}_{D}^{*}$. Because $\cup \mathcal{B}_{D}=V(D)$, $\mathcal{B}_{D}^{*}$ is referred to as the minimal complete cover of $\left(D, \tau_{D}\right)$ according to the base $\mathcal{B}_{D}$.

We will define a pseudo-metric map on graph $D$.

Definition2.7. Let $D=(V(D), E(D))$ be a nonempty graph, then $d: V(D) \times V(D) \rightarrow[0,+\infty)$ is called pseudo-metric map on $D$, if for all $r, v, u \in V(D)$,

(a) $d(\mathrm{r}, \mathrm{r})=0$,

(b) $d(\mathrm{r}, v)=d(v, \mathrm{r})$,

(c) $d(\mathrm{r}, v) \leq d(\mathrm{r}, u)+d(u, v)$.

For each $r \in V(D), Q \subseteq D, \epsilon>0$,

$$
B(\mathrm{r}, \epsilon)=\{v \in V(D): d(r, v)<\epsilon\}, d(\mathrm{r}, Q)=\inf \{d(\mathrm{r}, v): v \in V(Q)\} .
$$

If there exists pseudo-metric mapdon $D$ where $\{B(\mathrm{f}, \epsilon): \mathrm{r} \in V(D), \epsilon>0\}$ configures a base of $D$, then a topological space $\left(D, \tau_{D}\right)$ is referred to as pseudo-metrizable space.

Proposition 2.8. Let $D$ be pseudo-metrizable space. If $Q \subseteq D$ and $d$ is pseudo-metric map on $D$, then $r \in \bar{Q}$ if and only if $d(r, Q)=0$.

Proof. $\mathrm{f} \in \bar{Q}$ if and only if for each $\epsilon>0, B(\mathrm{f}, \epsilon) \cap V(Q) \neq \emptyset$ if and only if for each $\epsilon>0$, there exists $u \in V(Q)$ such that $d(f, u)<\epsilon$ if and only if $\inf \{d(f, u): u \in V(Q)\}$ if and only if $d(\mathrm{r}, Q)=0$. 
Definition 2.9. Let $\left(D, \tau_{D}\right)$ be a topological space. $D$ is named a pseudo-discrete space if $Q \subseteq D$ is open in $D$ if and only if $Q$ is closed in $D$.

\section{The Properties of Topological Spaces Induced by a Reflexive Graph}

We will study through this part, the properties of the topological space $\left(D, \tau_{D}\right)$, where $\left(D, \tau_{D}\right)$ is produced by a reflexive graph $D$.

Lemma3.1. Let $D_{\alpha}$ be a reflexive graph and $D_{\beta}$ the transmitting expression of $D_{\alpha}$, for every $\mathrm{r} \in V(D)$, chose $L_{\mathrm{r}}=\left\{v \in V(D):(\mathrm{r}, v) \in E\left(D_{\beta}\right)\right\}$, then

(1) $L_{\mathrm{r}} \in \tau_{D_{\alpha}}$,

(2) $\left\{L_{r}\right\}$ is an open neighborhood base of $r$,

(3) $L_{\mathrm{r}}$ is compact subset of $\left(D_{\alpha}, \tau_{D_{\alpha}}\right)$,

(4) $\mathcal{B}_{D_{\alpha}}=\left\{L_{\mathrm{f}}: \mathrm{f} \in V(D)\right\}$ is a base for $\left(D_{\alpha}, \tau_{D_{\alpha}}\right)$.

Proof. (1) It is sufficient to show that $L_{\mathrm{r}} \subseteq \operatorname{Int}\left(L_{\mathrm{r}}\right)$. Let $u \in L_{\mathrm{r}}$, so $(\mathrm{f}, u) \in E\left(D_{\beta}\right)$, then there exists $\left\{v_{1}, v_{2}, v_{3}, \ldots, v_{n}\right\} \subseteq V(D)$ where $\left(\mathrm{r}, v_{1}\right) \in E\left(D_{\alpha}\right),\left(v_{1}, v_{2}\right) \in E\left(D_{\alpha}\right), \ldots,\left(v_{n}, u\right) \in$ $E\left(D_{\alpha}\right)$, so $u \in\left[v_{n}\right]_{D_{\alpha}}$. For $y \in V(D)$ such that $y \in L_{\mathrm{f}},\left(v_{n}, y\right) \in E\left(D_{\alpha}\right)$, $\operatorname{so}\left(\mathrm{f}, v_{1}\right) \in E\left(D_{\alpha}\right)$, $\left(v_{1}, v_{2}\right) \in E\left(D_{\alpha}\right), \ldots,\left(v_{n}, y\right) \in E\left(D_{\alpha}\right)$, then $(\mathrm{f}, y) \in E\left(D_{\beta}\right)$, so $y \in[\mathrm{r}]_{D_{\beta}}$, then $y \in L_{\mathrm{r}}$, $\left[v_{n}\right]_{D_{\alpha}} \subseteq L_{\mathrm{r}}$, sou $\in \operatorname{Int}\left(L_{\mathrm{r}}\right)$, which implies to $L_{\mathrm{r}} \subseteq \operatorname{Int}\left(L_{\mathrm{r}}\right)$. Hence, $L_{\mathrm{r}} \in \tau_{D_{\alpha}}$.

(2) Let $B \in \tau_{D}$ such that $\mathrm{r} \in B$, we will show that $L_{\mathrm{r}} \subseteq B$. Let $u \in L_{\mathrm{f}}$, then $(\mathrm{f}, u) \in E\left(D_{\alpha}\right)$ or there exists $\left\{v_{1}, v_{2}, v_{3}, \ldots, v_{n}\right\} \subseteq V(D)$ where $\left(\mathrm{f}, v_{1}\right) \in E\left(D_{\alpha}\right),\left(v_{1}, v_{2}\right) \in E\left(D_{\alpha}\right), \ldots$, $\left(v_{n}, u\right) \in E\left(D_{\alpha}\right)$.

(i) If $(\mathrm{r}, u) \in E\left(D_{\alpha}\right)$, then we claim $u \in B$. For otherwise, $u \in B^{c}$, then, $L_{\mathrm{r}} \subseteq B^{c}$, but $D_{\alpha}$ is reflexive, so $\mathrm{r} \in L_{\mathrm{r}}$ and $L_{\mathrm{r}} \subseteq B^{c}$, then $\mathrm{r} \in B^{c}$, which is a contradiction. Hence, $u \in B$.

(ii) If there exists $\left\{v_{1}, v_{2}, v_{3}, \ldots, v_{n}\right\} \subseteq V(D)$ where $\left(\mathrm{f}, v_{1}\right) \in E\left(D_{\alpha}\right),\left(v_{1}, v_{2}\right) \in E\left(D_{\alpha}\right), \ldots$, $\left(v_{n}, u\right) \in E\left(D_{\alpha}\right)$, then by (i) we get $v_{1} \in B, v_{2} \in B, \ldots, u \in B$.

So $L_{\mathrm{r}} \subseteq B$, Which implies to $\left\{L_{\mathrm{r}}\right\}$ constitutes an open neighborhood base of $\mathrm{r}$.

(3) Let $\left\{K_{\lambda} \mid \lambda \in \Lambda\right\}$ be an open cover of $L_{\mathrm{r}}$ then $\mathrm{r} \in K_{\lambda_{i}}$ for some $\lambda_{i} \in \Lambda$, then by (2) $L_{\mathrm{r}} \subseteq K_{\lambda_{i}}$. Therefore, $L_{\mathrm{r}}$ is a compact subset of $\left(D, \tau_{D}\right)$.

(4) It is obvious by (2)

Remark 3.2. (1) Let $D=(V(D), E(D))$ be a graph, for each $\mathrm{r}, u \in V(D)$, if $(\mathrm{f}, u) \in$ $E(D)$ and $(u, \mathrm{r}) \in E(D)$, then $L_{\mathrm{r}}=L_{u}$.

(2) For all $B \in \mathcal{B}_{D}, B$ cannot be represented as the union of some elements of $\mathcal{B}_{D} \backslash\{B\}$. Otherwise, there exists $\mathcal{O}_{D} \subseteq \mathcal{B}_{D} \backslash\{B\}$ such that $B=\cup \mathcal{O}_{D}$. By $B \in \mathcal{B}_{D}$, there exists $\mathrm{f} \in V(D)$ where $B=L_{\mathrm{r}}$. Because $\mathrm{f} \in B$, there exists $Q \in \mathcal{O}_{D}$ such that $\mathrm{f} \in Q \subseteq B$. By Lemma 3.1, $L_{\mathrm{r}} \subseteq Q$. Then $B=Q$, so we obtain a contradiction. Hence, $H$ cannot be represented as the union of some elements of $\mathcal{B}_{D} \backslash\{B\}$. 
(3) Let $\mathcal{H}_{D}$ form a base for $\left(D, \tau_{D}\right)$. Then $\mathcal{B}_{D} \subseteq \mathcal{H}_{D}$. Otherwise, there exists $B \in \mathcal{B}_{D}$ but $B \notin \mathcal{H}_{D}$. Notice that $B \in \mathcal{B}_{D}$, there exists $\mathrm{f} \in B$ where $B=L_{\mathrm{r}}$. Because $\mathcal{H}_{D}$ is a base for $\left(D, \tau_{D}\right)$, there exists $\mathcal{H}_{D}^{l} \subseteq \mathcal{H}_{D}$ such that $B=\cup \mathcal{H}_{D}^{l}$. Thus $\mathrm{f} \in H \subseteq B$ for some $H \in \mathcal{H}_{D}^{l}$. By using Lemma 3.1, $B \subseteq H$. So $B=H \in \mathcal{H}_{D}$ and that means a contradiction. Therefore, $\mathcal{B}_{D} \subseteq$ $\mathcal{H}_{D}$.

Theorem 3.3.Let $\left(D, \tau_{D}\right)$ be a topological space generated by a reflexive graph $D$, then

(1) $\left(D, \tau_{D}\right)$ is a first countable space,

(2) $\left(D, \tau_{D}\right)$ is a locally compact space,

(3) If $D$ is countable, then $\left(D, \tau_{D}\right)$ is second countable space.

Proof. (1) By lemma 3.1(2) $\left\{L_{\mathrm{r}}\right\}$ is an open neighborhood base of $\mathrm{r}$, then $\left(D, \tau_{D}\right)$ is a first countable space.

(2) By lemma 3.1(3), we have for each $\mathrm{f} \in V(D)$, $\mathrm{r}$ has compact neighborhood. Hence $\left(D, \tau_{D}\right)$ is locally compact space.

(3) By lemma 3.1(4), $\mathcal{B}_{D}=\left\{L_{\mathrm{f}}: \mathrm{f} \in V(D)\right\}$ is a base for $\left(D, \tau_{D}\right)$, which implies to there exists a countable base for $\tau_{D}$, so $\left(D, \tau_{D}\right)$ is a second countable space.

Theorem 3.4. If $D=(V(D), E(D))$ is a reflexive graph and $D_{\beta}$ the transmitting expression of $D$, then $\left(D, \tau_{D}\right)=\left(D, \tau_{D_{\beta}}\right)$.

Proof. According to Lemma 3.1(4), $\mathcal{B}_{D}=\left\{L_{\mathrm{r}}: \mathrm{r} \in V(D)\right\}$ is a base for $\left(D, \tau_{D}\right)$, we will prove that $\mathcal{B}_{D}=\left\{L_{\mathrm{r}}: \mp \in V(D)\right\}$ is also a base for $\left(D, \tau_{D_{\beta}}\right)$.

By definition $L_{\mathrm{r}}=\left\{u V(D):(\mathrm{r}, u) \in D_{\beta}\right\} \in \tau_{D_{\beta}}$. Let $\mathrm{r} \in K \in \tau_{D_{\beta}}$, for any $u \in L_{\mathrm{r}}$, then $(\mathrm{r}, u) \in D_{\beta}$, since $\mathrm{r} \in K$, by Lemma 3.1(2) $\mathrm{r} \in L_{\mathrm{r}} \subseteq K$. So $\mathcal{B}_{D}$ is also base for $\tau_{D_{\beta}}$, hence $\left(D, \tau_{D}\right)=\left(D, \tau_{D_{\beta}}\right)$.

Lemma 3.5. Let $\left(D, \tau_{D}\right)$ be atopological space generated by a reflexive graph $D$, if $\mathcal{B}_{D}^{*}$ the minimal complete cover of $\left(D, \tau_{D}\right)$ according to the base $\mathcal{B}_{D}$, then for all $F \in \mathcal{B}_{D}^{*}, \mathrm{U}\left(\mathcal{B}_{D} \backslash\right.$ $\{F\}) \neq V(D)$ and $U\left(\mathcal{B}_{D}^{*} \backslash\{F\}\right) \neq V(D)$.

Proof. Suppose that $U\left(\mathcal{B}_{D} \backslash\{F\}\right)=V(D)$, then there exists $\mathcal{B}_{D}^{\iota} \subseteq \mathcal{B}_{D} \backslash\{F\}$ such that $F \subseteq \cup \mathcal{B}_{D}^{l}$, Since $F \in \mathcal{B}_{D}^{*} \subseteq \mathcal{B}_{D}$, there exists $\mathrm{r} \in V(D)$ such that $F=L_{\mathrm{f}}$, so $\mathrm{r} \in F^{\iota}$ for some $F^{\iota} \in \mathcal{B}_{D}^{\iota}$. By Lemma 3.1(2), $F=L_{\mathrm{r}} \subseteq F^{\iota}$. Consequently $F$ is not a maximal element of $\mathcal{H}_{G}$ which implies a contradiction. So, $\bigcup\left(\mathcal{B}_{D} \backslash\{F\}\right) \neq V(D)$. Since $U\left(\mathcal{B}_{D} \backslash\{F\}\right) \neq V(D), \cup\left(\mathcal{B}_{D}^{*} \backslash\{F\}\right) \neq V(D)$.

Lemma 3.6.Let $\left(D, \tau_{D}\right)$ be a topological space generated by a reflexive graph $D$, If $\mathcal{B}_{D}^{*}$ the minimal complete cover of $\left(D, \tau_{D}\right)$ according to the base $\mathcal{B}_{D}$ and $\mathcal{H}_{D}$ an open cover of $\left(D, \tau_{D}\right)$. Then for all $F \in \mathcal{B}_{D}^{*}$, there exists $H \in \mathcal{H}_{D}$ where $F \subseteq H$.

Proof. Since $\mathcal{H}_{D}$ an open cover of $\left(D, \tau_{D}\right)$, for any $F \in \mathcal{B}_{D}^{*}$, there exists $\mathcal{H}_{D}^{l} \subseteq \mathcal{H}_{D}$ such that $F \subseteq \cup \mathcal{H}_{D}^{l}$. Because $F \in \mathcal{B}_{D}^{*} \subseteq \mathcal{B}_{D}$, then $F=L_{\mathrm{r}}$ for some $\mathrm{r} \in F$, so there exists $H \in \mathcal{H}_{D}^{l} \subseteq$ $\mathcal{H}_{D}$ such that $\mathrm{r} \in H$. By Lemma $3.1, F \subseteq H$. 
Lemma 3.7.Let $\left(D, \tau_{D}\right)$ be a topological space generated by a reflexive graph $D$. If $\mathcal{B}_{D}^{*}$ is the minimal complete cover of $\left(D, \tau_{D}\right)$ according to the base $\mathcal{B}_{D}$ and $\mathcal{H}_{D}$ an open cover of $\left(D, \tau_{D}\right)$, which is made up of some elements of $\mathcal{B}_{D}$, then $\mathcal{B}_{D}^{*} \subseteq \mathcal{O}_{D}$.

Proof. For each $B \in \mathcal{B}_{D}^{*}$, we claim that $B \in \mathcal{O}_{D}$. If not, $B \notin \mathcal{O}_{D}$. Since $\cup \mathcal{O}_{D}=V(D), \cup\left(\mathcal{O}_{D} \backslash\right.$ $\{B\})=V(D)$, So $\cup\left(\mathcal{B}_{D} \backslash\{B\}\right)=V(D)$. By using Lemma3.5, $\cup\left(\mathcal{B}_{D} \backslash\{B\}\right) \neq V(D)$, which implies a contradiction. So $\mathcal{B}_{D}^{*} \subseteq \mathcal{O}_{D}$.

Theorem 3.8.Let $\left(D, \tau_{D}\right)$ be a topological space generated by a reflexive graph $D, \mathcal{B}_{D}^{*}$ the minimal complete cover of $\left(D, \tau_{D}\right)$ according to the base $\mathcal{B}_{D}$. Then $\mathcal{H}_{G}^{*}$ is a finite set if and only $\operatorname{if}\left(D, \tau_{D}\right)$ is compact space.

Proof. The only if part clear by Lemma (4.6).Conversely, suppose that $\left(D, \tau_{D}\right)$ is compact, as $\mathcal{H}_{G}$ is an open cover of $\left(D, \tau_{D}\right)$ then $\mathcal{H}_{G}$ has a finite subcover $\mathcal{H}_{G}^{\iota}$. By using Lemma 3.7, $\mathcal{B}_{D}^{*} \subseteq \mathcal{B}_{D}^{\iota}$, thus $\left|\mathcal{B}_{D}^{*}\right| \leq\left|\mathcal{B}_{D}^{\iota}\right|$. Hence $\mathcal{B}_{D}^{*}$ is a finite set.

\section{The Properties of Topological Spaces generated by a Tolerance Graph}

Through this part, we will achieve the properties of $\left(D, \tau_{D}\right)$, where $\left(D, \tau_{D}\right)$ is a topological space induced by tolerance graph $D$.

Lemma 4.1If $\left(D, \tau_{D}\right)$ is a topological space generated by a tolerance graph $D$, then for all $Q \subseteq D, Q$ is open if and only if $Q$ is closed.

Proof. $Q$ is open $\Leftrightarrow Q=\operatorname{Int}(Q) \Leftrightarrow Q^{c}=\operatorname{Int}\left(Q^{c}\right) \Leftrightarrow Q^{c}$ is open graph $\Leftrightarrow Q$ is closed.

Theorem 4.2. If $\left(D, \tau_{D}\right)$ is a topological space generated by a tolerance graph $D$. Then $\left(D, \tau_{D}\right)$ is discrete if and only if $\left(D, \tau_{D}\right)$ is $T_{0}$ - space.

Proof.The only if part is clear. We are going to prove the if part. Let $\left(D, \tau_{D}\right)$ be $T_{0}-$ space. Depending on the Lemma 3.1(4), we have if $D$ is reflexive, then $\left\{L_{\mathrm{r}}: \mathrm{r} \in V(D)\right\}$ is a base for $\left(D, \tau_{D}\right)$. We claim that $L_{\mathrm{r}}=\{\mathrm{r}\}$ for any $\mathrm{r} \in V(D)$. Suppose that $L_{\mathrm{r}} \neq\{\mathrm{r}\}$ for some $\mathrm{r} \in V(D)$. By Proposition $2.5, D_{\beta}$ is an equivalent graph on $V(D)$, so $L_{\mathrm{r}}=[\mathrm{r}]_{D_{\beta}}$. Chose $u \in[\mathrm{r}]_{D_{\beta}}$ such that $u \neq \mathrm{r}$. Since $\left(D, \tau_{D}\right)$ is $T_{0}-$ space, there exists an open subgraph $O$ where $\mathrm{r} \in V(O)$ and $u \notin V(O)$, or there exists an open subgraph $U$ where $u \in V(U)$ and $\mathrm{f} \notin V(U)$. If there exists an open subgraph $V(O)$ where $\mathrm{r} \in V(O)$ and $\mathrm{r} \notin V(U)$, then $\mathrm{r} \in L_{v} \subseteq V(O)$ for some $v \in$ $V(D)$ depending on the Lemma 3.1(4). It follows $u \notin L_{v}$. As $D_{\beta}$ is an equivalence graph on $V(D),[\mathrm{r}]_{D_{\beta}}=[v]_{D_{\beta}}=L_{v}$. Thus $u \in[\mathrm{r}]_{D_{\beta}}=L_{v}$ means a contradiction. Similarly if there exists an open subgraph $U$ where $u \in V(U)$ and $\mathrm{r} \notin V(U)$. Hence, $\{\mathrm{r}\}$ is open for all $\mathrm{r} \in V(D)$. Therefore, all subgraphs of $V(D)$ are open which means that $\left(D, \tau_{D}\right)$ is discrete.

Theorem 4.3.Let $\left(D, \tau_{D}\right)$ be a topological space generated by a tolerance graph $D$. Then, the statements are equivalent:

(1) $V(D) / E\left(D_{\beta}\right)$ is countable,

(2) $\left(D, \tau_{D}\right)$ is a second countable space,

(3) $\left(D, \tau_{D}\right)$ is a separable space, 
(4) $\left(D, \tau_{D}\right)$ is a lindelöf space.

Proof. $(1) \Longrightarrow(2)$. Since $D_{\beta}$ is an equivalence graph on $V(D),\left\{L_{\mathrm{r}}: \mathrm{f} \in V(D)\right\}=V(D) / E\left(D_{\beta}\right)$. By Lemma 3.1(4), $\left(D, \tau_{D}\right)$ is second countable space.

$(2) \Longrightarrow(1)$ Suppose that $\mathcal{B}$ is a countable base for $\left(D, \tau_{D}\right)$, then for $r \in V(D)$, there exists $B_{\mathrm{r}} \in \mathcal{B}$ such that $\mathrm{r} \in B_{\mathrm{r}} \subseteq L_{\mathrm{r}}$. By Lemma 3.1(4), $\mathrm{r} \in L_{u} \subseteq B_{\mathrm{r}}$ for some $u \in V(D)$. Since $L_{\mathrm{r}}=[\mathrm{r}]_{D_{\beta}}=[u]_{D_{\beta}}=L_{u}, B_{\mathrm{r}}=[\mathrm{r}]_{D_{\beta}}$, we define $f: V(D) / E\left(D_{\beta}\right) \rightarrow \mathcal{B}$ by $f\left([\mathrm{r}]_{D_{\beta}}\right)=B_{\mathrm{r}}$, then $f$ is injective. So $\left|V(D) / E\left(D_{\beta}\right)\right| \leq|\mathcal{B}|$. Hence $V(D) / E\left(D_{\beta}\right)$ is countable.

$(2) \Longrightarrow(3)$ and $(2) \Rightarrow(4)$ are clear.

(3) $\Rightarrow$ (2). Suppose that $C$ is a countable dense subgraph of $\left(D, \tau_{D}\right)$. Put $\lambda=\left\{L_{\mathrm{f}}: \mathrm{f} \in V(C)\right\}$, then $\lambda$ is countable. By Lemma 3.1(4), for all $\mathrm{f} \in V(D)$ and open subgraph $O$ with $\mathrm{r} \in V(O)$, we have $\mathrm{r} \in L_{u} \subseteq V(O)$ for some $u \in V(D)$. Since $C$ is dense, $L_{u} \cap V(C) \neq \varnothing$, Chose $v \in L_{u} \cap V(C)$, then $L_{v} \in \lambda$. Since $D_{\beta}$ is an equivalence graph on $V(D), L_{v}=[v]_{D_{\beta}}=$ $[u]_{D_{\beta}}=L_{u}$. It follows $\mathrm{r} \in L_{v} \subseteq V(O)$. Therefore, $\lambda$ is a base for $\left(D, \tau_{D}\right)$. Hence $\left(D, \tau_{D}\right)$ is a second countable space.

(4) $\Longrightarrow$ (2). Suppose that $V(D) / E\left(D_{\beta}\right)$ is not countable. Since $D_{\beta}$ is an equivalence graph on

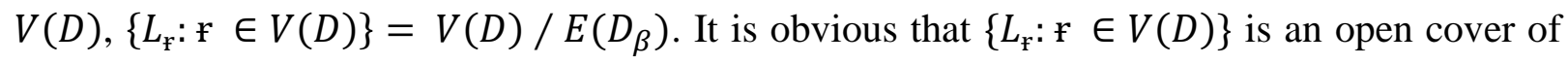
$\left(D, \tau_{D}\right)$ but $\left\{L_{\mathrm{r}}: \mathrm{r} \in V(D)\right\}$ does not have any countable subcover Hence we get a contradiction.

Theorem 5.4 Let $\left(D, \tau_{D}\right)$ be a topological space generated by a tolerance graph $D$. Then $\left(D, \tau_{D}\right)$ is a connected space if and only if $E\left(D_{\beta}\right)=V(D) \times V(D)$.

Proof. Suppose that $\left(D, \tau_{D}\right)$ is connected, If $E\left(D_{\beta}\right) \neq V(D) \times V(D)$, then $V(D) \times V(D) /$ $E\left(D_{\beta}\right) \neq \emptyset$. Chose $(\mathrm{r}, u) \in(V(D) \times V(D)) \backslash E\left(D_{\beta}\right)$, then $u \notin[\mathrm{r}]_{D_{\beta}}=L_{\mathrm{r}}$. So $L_{\mathrm{r}} \neq V(D)$ and $L_{\mathrm{r}} \neq \varnothing$. By Lemma $4.1, L_{\mathrm{r}}$ is both open and closed, so we obtain a contradiction. Conversely, Suppose that $E\left(D_{\beta}\right)=V(D) \times V(D)$, then $V(D) / E\left(D_{\beta}\right)=[V(D)]$. So $\tau_{D}=$ $\{V(D), \emptyset\}$, thus $\left(D, \tau_{D}\right)$ is connected.

Theorem 4.6. Let $\left(D, \tau_{D}\right)$ be a topological space generated by a tolerance graph $D$. Then

(1) $\left(D, \tau_{D}\right)$ is a locally connected space

(2) $\left(D, \tau_{D}\right)$ is a locally separable space,

(3) $\left(D, \tau_{D}\right)$ is a regular space,

(4) $\left(D, \tau_{D}\right)$ is a normal space,

(5) $\left(D, \tau_{D}\right)$ is a pseudo-metrizable space.

Proof.(1) By lemma 3.1(2) every open neighborhood of $\mathbf{r}$ contains $L_{\mathrm{r}}$ which is connected.

(2)Since $\left\{L_{\mathrm{r}}\right\}$ is an open neighborhood base of $\mathrm{r}$, we just need to show that $L_{\mathrm{r}}$ is a separable subset of $\left(D, \tau_{D}\right)$. Let $\overline{\{\mathrm{r}\}}$ be the closure of $\{\mathrm{r}\}$ and suppose that there exists $u \in \overline{\{\mathrm{F}\}}$ such that $u \notin L_{\mathrm{r}}$, so $[\mathrm{r}]_{D_{\beta}} \cap[u]_{D_{\beta}}=\emptyset$. For an open neighborhood $L_{u}$ of $u,\{\mathrm{r}\} \cap L_{u}=\emptyset$, so $u \notin \overline{\{\mathrm{r}\}}$ 
which is a contradiction, hence, $u \in L_{\mathrm{r}}$ then $\overline{\{\mathrm{r}\}} \subseteq L_{\mathrm{r}}$. On the other hand, let $u \in L_{\mathrm{r}}$ then $u \in[\mathrm{r}]_{D_{\beta}}$, then $L_{\mathrm{r}}=L_{u}$. Suppose $O$ is an open neighborhood of $u$, so $L_{u} \subseteq V(O)$ then $L_{u} \cap V(O) \neq \varnothing$, so $L_{\mathrm{r}} \cap V(O) \neq \varnothing$, then, $\{\mathrm{f}\} \cap V(O) \neq \emptyset$ then $u \in \overline{\{\mathrm{f}\}}$, so $L_{\mathrm{r}} \subseteq \overline{\{\mathrm{f}\}}$. Hence, $L_{\mathrm{r}}=\overline{\{\mathrm{r}\}}$, and we obtained that $\{\mathrm{r}\}$ is countable dense subset of $L_{\mathrm{r}}$ which implies to $L_{\mathrm{r}}$ is separable subset of $\left(D, \tau_{D}\right)$. Hence, $\left(D, \tau_{D}\right)$ is locally separable space.

(3) Let $Q$ be closed subgraph of $D$ and $\mathrm{f} \in V(Q)^{c}$, by Lemma $4.1 Q$ is open if and only if $Q$ is closed, so $Q$ and $Q^{c}$ are two open disjoint subgraph of $D$ such that $V(Q) \subseteq V(Q)$ and $\mathrm{r} \in$ $V(Q)^{c}$. Hence $\left(D, \tau_{D}\right)$ is a regular space.

(4) Let $Q, M$ are two disjoint closed subgraphs of $D$, then by Lemma 4.1 they are also disjoint closed subgraphs of $V(D)$. But we have $V(Q) \subseteq V(Q)$ and $V(M) \subseteq V(M)$. Hence $\left(D, \tau_{D}\right)$ is a normal space.

(5) Since there exists the trivial pseudo-metrizable map $d$ induced by the pseudo-metrizable space, where

$$
d: V(D) \times V(D) \rightarrow[0, \infty), \text { such that } d= \begin{cases}1 & \text { if } \mathrm{r}=u \\ 0 & \text { if } \mathrm{r} \neq u\end{cases}
$$

For any $\mathrm{f} \in V(D)$ and $\epsilon>0$,

$$
B(\mathrm{r}, \epsilon)= \begin{cases}\{\mathrm{r}\} & \text { if } \epsilon<1 \\ V(D) & \text { if } \epsilon \geq 1\end{cases}
$$

Then, $\{\mathrm{f}\} \in \tau_{D}$, so $\left(D, \tau_{D}\right)$ is pseudo-discrete, then $B(\mathrm{f}, 1)=\{u \in V(D): d(\mathrm{f}, u)<1\}=$ $\{u \in V(D): d(\mathrm{f}, u)=0\}=\{\mathrm{r}\}$. Thus $\{B(\mathrm{f}, \epsilon): \mathrm{r} \in V(D)$ and $\epsilon>0\}$ forms a base for $\left(D, \tau_{D}\right)$. Hence $\left(D, \tau_{D}\right)$ is pseudo-metrizable.

\section{Approximation spaces on digraph}

We will present the concept of approximation spaces in this part; furthermore, we will get their characterizations and properties.

Definition 5.1. Let $(D, \rho)$ be a topological space, then $(D, \rho)$ is called an approximation space if there exists an equivalence graph $D=(V(D), E(D))$ such that $\tau_{D}=\rho$.

According to Lemma 4.1, we get that approximating spaces are pseudo-discrete spaces. But the question, would pseudo-discrete spaces are approximating space? This problem is certainly answered by the following theorem.

Theorem 5.2. 6.2 If $(D, \rho)$ is a topological space, we have the next equivalence:

(1) $(D, \rho)$ is an approximating space,

(2) $(D, \rho)$ is both pseudo-metrizable and pseudo-discrete,

(3) $(D, \rho)$ is pseudo-discrete space.

Proof. $(1) \Rightarrow(2)$. It holds depending on Lemma 4.1 and Theorem 4.6. 
$(2) \Rightarrow(1)$. Let $(D, \rho)$ be both pseudo-metrizable and pseudo-discrete, then there exists a pseudo-metric map $d$ on $V(D)$ where $\{B(f, \epsilon): f \in V(D)$ and $\epsilon>0\}$ is a base for $(D, \rho)$. We define a graph $D$ on $V(D)$ as thereinafter:

$$
\text { For all } r, u \in V(D),(r, u) \in E(D) \text { if and only if } d(r, u)=0 \text {. }
$$

Since $d$ is pseudo-metric on $V(D)$, so $D$ is an equivalence graph. We will prove that $\tau_{D}=\rho$. Let $Q \in \rho$, by Proposition 2.8, $\bar{Q}=\{\mathrm{r} \in V(D): d(\mathrm{r}, Q)=0\}$. Since $(D, \rho)$ is pseudo-discrete, $Q$ is closed in $(D, \rho)$, so $V(Q)=\{\mathrm{f} \in V(D): d(\mathrm{r}, Q)=0\}$. It is obvious that $V(Q) \subseteq$ $U\left\{[\mathrm{r}]_{D}: \mathrm{r} \in V(Q)\right\}$. If $u \in[\mathrm{r}]_{D}$ with $\mathrm{r} \in V(Q)$, then, $d(\mathrm{f}, Q) \leq d(u, \mathrm{f})=0$. So $u \in$ $\{\mathrm{r} \in V(D): d(\mathrm{r}, Q)=0\}=V(Q)$, that is mean $V(Q) \supseteq \bigcup\left\{[\mathrm{r}]_{D}: \mathrm{r} \in V(Q)\right\}$. Thus $V(Q)=$ $\mathrm{U}\left\{[\mathrm{r}]_{D}: \mathrm{r} \in V(Q)\right\}$, it follows that $V(Q) \in \tau_{D}$, so $\rho \subseteq \tau_{D}$.

On the other side, let $\mathrm{f} \in V(D)$, by Proposition $2.8, \overline{\{\mathrm{f}\}}=\{u \in V(D): d(u, \mathrm{f})=0\}$, then $\overline{\{\mathrm{r}\}}=\{\mathrm{r}\}_{D}$. Now $[\mathrm{r}]_{D}$ is closed in $(D, \rho)$, since $(D, \rho)$ is pseudo-discrete, $[\mathrm{r}]_{D} \in \rho$. Since $\left\{[\mathrm{r}]_{D}: \mathrm{r} \in V(D)\right\}$ is a base for $\left(D, \tau_{D}\right), \tau_{D} \subseteq \rho$.

Hence $\tau_{D}=\rho$. This means that $(D, \rho)$ are an approximation space.

(2) $\Rightarrow(3)$. Clear.

(3) $\Rightarrow$ (2). Let $(D, \rho)$ be pseudo-discrete. For each $f \in V(D), C(f)$ denoted a connected component with $\mathrm{r} \in C(\mathrm{f})$, then $C(\mathrm{r})$ is closed in $(D, \rho)$. So $\overline{\{\mathrm{r}\}} \subseteq C(\mathrm{f})$. Let $u \in C(\mathrm{r})$, since $C(\mathrm{r})$ is a connected component with $\mathrm{r} \in C(\mathrm{r})$, there exists a connected subgraph $Q$ of $D$ wheref, $u \in V(Q)$. Since $D$ is pseudo-discrete, $\overline{\{\mathrm{r}\}}$ is both open and closed in $(D, \rho)$. Note that $\overline{\{\mathrm{F}\}} \cap V(Q)$ is both open and closed in the subspace $Q$ and $Q$ is connected. Then $\overline{\{\mathrm{r}\}} \cap V(Q)=$ $V(Q)$, so $u \in \overline{\{\mathrm{f}\}}$. This indicates that $C(\mathrm{f}) \subseteq \overline{\{\mathrm{f}\}}$. Thus $C(\mathrm{f})=\overline{\{\mathrm{f}\}}$.

We define $d: V(D) \times V(D) \rightarrow[0, \infty)$ as follows:

$$
d(\mathrm{r}, u)= \begin{cases}0 & \text { if } C(\mathrm{r})=C(u) \\ 1 & \text { if } C(\mathrm{r}) \neq C(u)\end{cases}
$$

The assumption that $d$ is pseudo-metric on $V(D)$ can be easily proved. For any $\mathrm{f} \in V(D)$ and $\epsilon>0$

$$
B(\mathrm{r}, \epsilon)= \begin{cases}\overline{\{\mathrm{f}\}} & \text { if } \epsilon \leq 1 \\ V(D) & \text { if } \epsilon>1\end{cases}
$$

Then $B(\mathrm{r}, \epsilon)$ will be closed in $(D, \rho)$. Because $D$ is pseudo-discrete, $B(\mathrm{f}, \epsilon) \in \rho$. Let $\mathrm{f} \in V(D)$ and $V(O) \in \rho$ with $\mathrm{r} \in V(Q)$. Since $D$ is pseudo-discrete, $V(Q)$ is closed in $(D, \rho)$. So $\overline{\{\mathrm{r}\}} \subseteq$ $V(Q)$. By Proposition 2.8, $\overline{\{\mathrm{F}\}}=\{u \in V(D): d(\mathrm{f}, u)=0\}$. Then.$V(D)$ and $\epsilon>0\}$ is a base for $(D, \rho)$. Therefore $(D, \rho)$ is pseudo-metrizable.

Corollary 5.3. Discrete spaces are approximating spaces.

Theorem 5.4. Quotient maps preserve approximating spaces. 
Proof. Suppose that the image of an approximating space Dunder a quotient map $f$ is $D^{\prime}$. We have to show that $D^{\prime}$ is an approximating space. Since $f$ is a quotient map, $N \subseteq D^{\prime}$ is open in $D^{\prime}$ if and only if $f^{-1}(N)$ is open in $D$. By using Theorem, 5.2, $f^{-1}(N)$ is open in $D$ if and only if $f^{-1}(N)$ is closed in $D$. Since $f$ is a quotient map, then, $f^{-1}(N)$ is closed if and only if $N \subseteq D^{\prime}$ is closed in $D^{\prime}$. So $N \subseteq D^{\prime}$ is open in $D^{\prime}$ if and only if $N$ is closed in $D^{\prime}$. According to Theorem $5.2, D^{\prime}$ is an approximating space.

Corollary5.5. Continuous maps do not preserve an approximating space.

We will explicate Corollary 5.5. in the next example.

Example 5.6.Suppose that $V(D)$ is a real numbers set $\mathrm{R}$ given with the usual discrete topology and $V\left(D^{\prime}\right)$ is a real numbers $R$ given with the usual Euclidean topology, let $f: V(D) \rightarrow V\left(D^{\prime}\right)$ be the identity map, it is obvious that $f$ is continuous map. According to the Corollary 5.3, $V(D)$ is an approximating space. But $V\left(D^{\prime}\right)$ is not an approximating space. Therefore, continuous maps do not preserve approximating spaces.

\section{Conclusion}

We offer the topological space generated by a reflexive graph and tolerance graph consecutively and discussed the topological structure of generalized rough graph. We have also achieving approximating spaces and get sufficient and necessary conditions that topological spaces are approximating spaces on graphs.

\section{References}

1. Wilson, R. J. Introduction of Graph Theory, Fourth, 1996.

2. Pawlak,Z.; Rough Sets, International Journal of Information and Computer Science, 1982 11,5, 341-356.

3. Li, Z. Topological properties of generalized rough sets, In: Proc. Seventh International Conference on Fuzzy Systems and Knowledge Discovery, 2010, 2067-2070.

4. Zhu, W. Topological approaches to coveringgener alizedroughsets, Information, 2007,177, 1499-1508.

5. Kondo, M. On the structure of generalized roughsets, Information Science, 2006, 176,589-600.

6. Engelking, R. GeneralTopology,PolishScientificPublishers, Warszawa,1977.

7. Obaid ,S. S. On Topological Structures in Graph Theory, master thesis, College of Education for Pure Science / Ibn Al-Haitham, University of Baghdad, 2017.

8. Yousif, Y. Y. Topological Generalizations of Rough Concepts, International Journal of Advanced Scientific and Technical Research, R S. Publication, 2015 , 5, 3, 265-272, May-June.

9. Abd El-Monsef, M. E.; Shokry, M. M.; Yousif Y. Y. Near Approximations in $\mathrm{G}_{\mathrm{m}^{-}}$ Closure Spaces, International Scholarly Research Network (ISRN) Applied Mathematics Volume 2012, Article ID 240315, 23 pages doi:10.5402/2012/240315, Hindawi Publishing Corporation, USA, 2012.

10. Shokry, M. ; Yousif, Y. Y. Closure Operators on Graphs, Australian Journal of Basic and Applied Sciences, Australian, 2011, 5, 11, 1856-1864. 
11. Shokry, M.; Yousif, Y. Y. Connectedness in Graphs and $\mathrm{G}_{\mathrm{m}}$-Closure Spaces, Journal of Computer Sciences, International Centre For Advance Studies, India, 2011, 22,3, 77 86.

12. Yousif, Y. Y.; Abdul-naby, A. I. Rough and Near Rough Probability in $\mathrm{G}_{\mathrm{m}}$-Closure Spaces, International Journal of Mathematics Trends and Technology, Seventh Sense Research Group, 2016, 30, 2, 68-78.

13. Yousif, Y. Y.; Obaid, S. S. Topological Structures Using Mixed Degree Systems in Graph Theory, International Journal of Applied Mathematics \& Statistical Sciences, International Academy of Sciences, 2016, 5, 2, 51-72.

14. Yousif, Y. Y. Topological Generalizations of Rough Concepts, International Journal of Advanced Scientific and Technical Research, $R$ S. Publication, 2015,5, 3, 265-272.

15. Radwan, A. E.; Yousif, Y. Y. Near Rough and Near Exact Subgraphs in $\mathrm{G}_{\mathrm{m}}$-Closure spaces, International Journal of Computer Science Issues (IJCSI), Mauritius, 2012, 9, 2, 3, 131-140.

16. Yousif, Y. Y.; Obaid S. S., Generalization of Rough Set Theory Using a Finite Number of a Finite d. g.'s, International Journal of Science and Research (IJSR), 2018,7,1,1043 -1052 . 